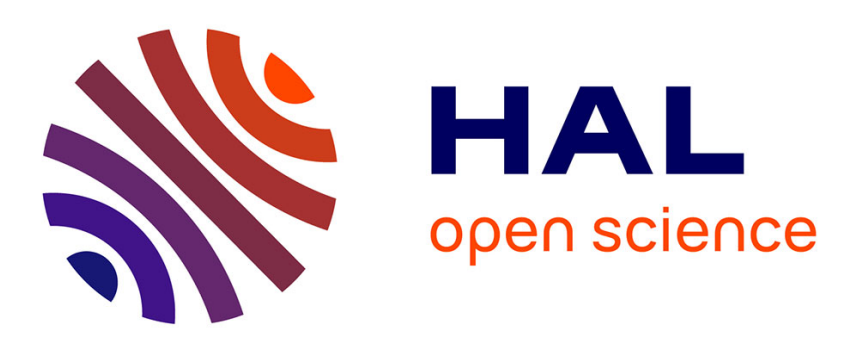

\title{
A non-local fuzzy segmentation method: Application to brain MRI
}

Benoît Caldairou, François Rousseau, Nicolas Passat, Piotr Habas, Colin

Studholme, Christian Heinrich

\section{To cite this version:}

Benoît Caldairou, François Rousseau, Nicolas Passat, Piotr Habas, Colin Studholme, et al.. A nonlocal fuzzy segmentation method: Application to brain MRI. International Conference on Computer Analysis of Images and Patterns (CAIP), 2009, Münster, Germany. pp.606-613, 10.1007/978-3-64203767-2_74. hal-01695014

\section{HAL Id: hal-01695014 \\ https://hal.univ-reims.fr/hal-01695014}

Submitted on 3 Mar 2018

HAL is a multi-disciplinary open access archive for the deposit and dissemination of scientific research documents, whether they are published or not. The documents may come from teaching and research institutions in France or abroad, or from public or private research centers.
L'archive ouverte pluridisciplinaire $\mathbf{H A L}$, est destinée au dépôt et à la diffusion de documents scientifiques de niveau recherche, publiés ou non, émanant des établissements d'enseignement et de recherche français ou étrangers, des laboratoires publics ou privés. 


\title{
A Non-Local Fuzzy Segmentation Method: Application to Brain MRI
}

\author{
B. Caldairou ${ }^{1}$, F. Rousseau ${ }^{1}$, N. Passat ${ }^{1}$, P. Habas ${ }^{2}$, C. Studholme ${ }^{2}$, \\ C. Heinrich ${ }^{1}$ \\ 1 LSIIT, UMR 7005 CNRS-Université de Strasbourg, Illkirch, 67412 France \\ 2 Biomedical Image Computing Group, University of California San Francisco, \\ San Francisco, CA 94143, USA
}

\begin{abstract}
The Fuzzy C-Means algorithm is a widely used and flexible approach for brain tissue segmentation from 3D MRI. Despite its recent enrichment by addition of a spatial dependency to its formulation, it remains quite sensitive to noise. In order to improve its reliability in noisy contexts, we propose a way to select the most suitable example regions for regularisation. This approach inspired by the Non-Local Mean strategy used in image restoration is based on the computation of weights modelling the grey-level similarity between the neighbourhoods being compared. Experiments were performed on MRI data and results illustrate the usefulness of the approach in the context of brain tissue classification.
\end{abstract}

Keywords: fuzzy clustering, regularisation, non-local processing, image segmentation, MRI.

\section{Introduction}

Segmentation methods of brain MRI can be categorised into 3 groups: classification methods, region-based methods and boundary-based methods. A very popular one is the K-mean algorithm which has been extended to fuzzy segmentation by Pham et al. in [10]. This so called Fuzzy C-Means (FCM) clustering algorithm is a powerful tool for MRI analysis since it authorises voxels to belong to several clusters with varying degrees of membership. Due to its flexibility, this segmentation framework has been intensively extended, for instance by including topological properties [2], DTI handling [1] or prior knowledge.

A main drawback of the standard FCM algorithm remains its sensitivity to noise in medical images. Many pixel-based regularisation term have been proposed such as Tikhonov regularisation [12], Markov Random Field (MRF), a priori image model or variational approaches. Inspired by works developed on MRF basics, Pham has proposed [9] a spatial model to improve the robustness to noise of FCM. However, the use of such pixel based regularisation terms assumes a specific image model: for instance, variational approaches can be based on the hypothesis that images are made of smooth regions separated by sharp edges. 
Recently, a non-local framework has been proposed to handle more efficiently repetitive structures and textures, for denoising purpose [4] or inverse problems $[3,8,11]$. In this work, we propose to introduce this non-local framework into the regularisation term of the FCM algorithm.

The sequel of this article is organised as follows. In Section 2, we present the segmentation problem and provide a short overview about FCM and regularisation. Section 3 details the new non-local approach for image segmentation. In Section 4, results obtained on the Brainweb database [5] are presented. Finally, Section 5 discusses these results and brings up to further work.

\section{Background}

\subsection{Fuzzy C-Means (FCM)}

The basics of this algorithm have been presented in [10] and make the segmentation equivalent to the minimisation of an energy function:

$$
J_{F C M}=\sum_{j \in \Omega} \sum_{k=1}^{C} u_{j k}^{q}\left\|\mathbf{y}_{j}-\mathbf{v}_{k}\right\|_{2}^{2} .
$$

This formulation is used to perform a $C$-classes segmentation. The parameter $u_{j k}$ represents the membership of the $k^{t h}$ class into the $j^{t h}$ voxel of the image, the parameter $q$ controls the "fuzziness" of the segmentation (if $q$ gets close to 1 , the segmentation becomes more crisp and close to a binary result), $\mathbf{v}_{k}$ represents the centroid of the $k^{t h}$ component and $\mathbf{y}_{j}$ represents the grey-level of the $j^{t h}$ voxel of the image. $\left\|\mathbf{y}_{j}-\mathbf{v}_{k}\right\|_{2}$ represents the Euclidean distance between the voxel's grey level and the considered centroid. The proportions are constrained so that: $\sum_{k=1}^{C} u_{j k}=1$.

Although this method has a fast convergence and provides reliable results in a convenient environment (low level of noise), the performance of this approach strongly decreases for noisy images. In such cases, anatomically hazardous structures may appear, for instance grey matter voxels among white matter volumes. However, FCM has shown to be easily extended and several approaches have been proposed in order to improve the robustness of FCM by introducing the idea of regularisation into the segmentation framework.

\subsection{Regularisation}

Regularisation is a classic method in inverse problem to determine the most accurate solution among many possible ones [12]. It introduces constraints to eliminate irrelevant solutions. In particular, Pham et al. in [9] added a regularisation term in Equation (1) to penalise unlikely configurations that can be met in the image. They called this method: Robust Fuzzy C-Means Algorithm (RFCM). The expression of the obtained energy function is then:

$$
J_{R F C M}=\sum_{j \in \Omega} \sum_{k=1}^{C} u_{j k}^{q}\left\|\mathbf{y}_{j}-\mathbf{v}_{k}\right\|_{2}^{2}+\frac{\beta}{2} \sum_{j \in \Omega} \sum_{k=1}^{C} u_{j k}^{q} \sum_{l \in N_{j}} \sum_{m \in M_{k}} u_{l m}^{q}
$$




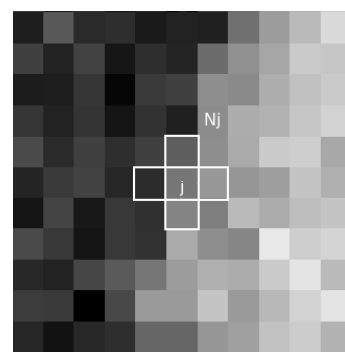

(a)

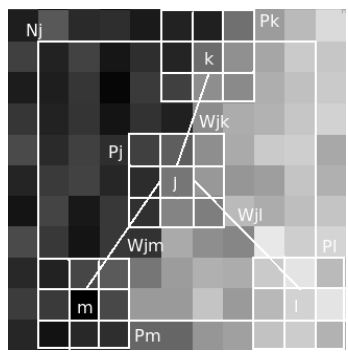

(b)

Fig. 1. Comparison of the RFCM [9] (a) and the NL approach (b). In this example, the area around the voxel $j$ is more similar to the one of voxel $k$ than the one of voxels $m$ and $l$. Therefore, the weight $w_{j k}$ will be higher than the weights $w_{j m}$ and $w_{j l}$.

where $N_{j}$ is the set of the neighbours of voxel $j$ and $M_{k}=\{1, \ldots, C\} \backslash\{k\}$. The new penalty term is minimised when the membership value for a particular class is large and the membership values for the other classes at neighbouring pixels are small (and vice versa).

The parameter $\beta$ controls the trade-off between the data-term and the smoothing term. Note that if $\beta=0$, we retrieve the classic FCM algorithm without any regularisation term. If $\beta>0$, the dependency on the neighbours causes $u_{j k}$ to be large when the neighbouring membership values of the other classes are small. The result is a smoothing effect that causes neighbouring membership values of a class to be negatively correlated with the membership values of the other classes. In [9], Pham et al. have proposed to estimate $\beta$ using cross-validation to obtain near-optimal performances, and they worked with a neighbourhood $\left(N_{j}\right)$ composed of the points 6 -adjacent to the current point $j$. In the work proposed hereafter, we focus on the use of a larger weighted neighbourhood relying on a non-local framework.

\section{Non-Local Regularisation}

\subsection{Non-Local Approach}

The Non-Local (NL) Regularisation is a strategy that has been proposed first as a denoising tool [4] and named as NL Mean denoising. Basically, it tries to take advantage of the redundancy of any natural image, broadly speaking a small neighbourhood around a voxel may match neighbourhoods around other voxels of the same image.

The non-local framework proposed by Buades et al. [4] relies on a weighted graph $w$ that links together voxels over the image domain. The computation of this graph $w$ is based on the similarity between neighbourhoods of voxels (see illustration in Fig. 1).

In the sequel, we will call such a neighbourhood a patch and denote the patch around voxel $j$ as $P_{j}$. The similarity of two voxels is defined as the similarity 
of the grey-levels contained into $P_{i}$ and $P_{j}$. This similarity can be computed as a Gaussian weighted Euclidean distance, but it has been shown that a simple Euclidean distance is reliable enough [6]. The weight for the voxels $i$ and $j$ is defined as follows:

$$
w_{i j}=\frac{1}{Z_{i}} e^{-\frac{\left\|\mathbf{y}\left(P_{i}\right)-\mathbf{y}\left(P_{j}\right)\right\|_{2}^{2}}{h^{2}}}
$$

where $Z_{i}$ is a normalisation constant and $h$ is a smoothing parameter. The distance between patches is defined as follows:

$$
\left\|\mathbf{y}\left(P_{i}\right)-\mathbf{y}\left(P_{j}\right)\right\|_{2}^{2}=\sum_{p=1}^{\left|P_{i}\right|}\left(y^{(p)}\left(P_{i}\right)-y^{(p)}\left(P_{j}\right)\right)^{2}
$$

where $\mathbf{y}\left(P_{i}\right)$ is the vector containing the grey-levels of the neighbourhood and $y^{(p)}\left(P_{i}\right)$ is the $p^{t h}$ component of this vector.

Note that it is possible to set the parameter $h$ automatically [6] by setting: $h^{2}=2 \alpha \sigma^{2}\left|P_{i}\right|$. The parameter $\sigma^{2}$, namely the standard deviation of the noise, can be computed directly from the image. If the noise in the image is Gaussian, we can set the parameter $\alpha$ to 1 [6]. Otherwise, it can be adjusted to get a more accurate result.

The NL Regularisation approach has already been successfully applied to different kinds of image processing problems. Mignotte [8] used this procedure to constrain a deconvolution process, Bougleux et al. [3] integrated it into the resolution of inverse problems and Rousseau [11] applied it for super-resolution reconstruction techniques.

\section{$3.2 \quad$ Non-Local Fuzzy C-Means Algorithm (NL-FCM)}

The key point of the NL approach is the capacity to handle a large neighbourhood without prior knowledge. We show in this work that such methodology can be easily introduced into the FCM framework. We investigate larger neighbourhoods to provide more information for the regularisation. Moreover, the underlying assumption is that voxels who have similar patches in the research area belong to the same tissue as shown in Fig. 1(b). We propose to define a NL version of FCM as follows:

$$
J_{N L-F C M}=\sum_{j \in \Omega} \sum_{k=1}^{C} u_{j k}^{q}\left\|\mathbf{y}_{j}-\mathbf{v}_{k}\right\|^{2}+\frac{\beta}{2} \sum_{j \in \Omega} \sum_{k=1}^{C} u_{j k}^{q} \sum_{l \in N_{j}} w_{j l} \sum_{m \in M_{k}} u_{l m}^{q} .
$$

Compared to Equation (2), a weight parameter is introduced in order to automatically balance the influence of voxels in the neighbourhood $N_{j}$. Note also that contrary to Pham et al. [9] where $N_{j}$ was a six-neighbourhood system, we investigate in this work larger neighbourhood systems such as the ones used in non-local denoising approach of Buades et al. [4].

The regularisation term of the energy function defined in Equation (5) takes into account the image content in an adaptive and flexible manner to smooth 
the current segmentation map. In other words, if two voxels neighbourhoods are similar, there might be a chance that they belong to the same tissue and so, the weight $w_{j l}$ increases. Conversely, if two voxels in the original image are quite different, it is normal to decrease the influence of the regularisation term since there is a lower probability that this voxel might have a good influence on the classification of the current one.

The proposed method (and the other ones considered for validation: FCM and RFCM) were optimised through Pham's way [9]: we used the same analytical expressions for the calculation of the centroids and of the membership functions.

\section{Results}

\subsection{Influence of the Non-Local Parameters $\left(\alpha, N_{j}\right)$}

Experiments have been carried out on simulated brain MRI images provided by the Brainweb database [5]. Notice that we perform a 3-class segmentation (Cerebro Spinal Fluid (CSF), Grey Matter (GM), White Matter (WM)) on a T1-weighted image corrupted by a $9 \%$ Rician noise (characteristic from MRI images) [7].

The Brainweb ground truth is used to assess the influence of parameters $\left(\alpha, N_{j}\right)$ of the proposed non-local method. In order to quantify the quality of the segmentation results, we use the following overlap measure:

$$
K I=\frac{2 . T P}{2 . T P+F P+F N}
$$

where $T P$ is the amount of true positives, $F P$ is the amount of false positives and $F N$, the amount of false negatives.

In this work, $N_{j}$ is considered as a cubic neighbourhood. Results for different sizes of $N_{j}$ (from $3 \times 3 \times 3$ up to $13 \times 13 \times 13$ voxels) are stated in Fig. 3(a). These experiments emphasise that considering extended neighbourhoods is a way to improve the segmentation results. Nevertheless, increasing $N_{j}$ above a $5 \times 5 \times 5$ size does not refine the segmentation results and slows the computation down. Therefore, we chose to run the validations in subsection 4.2 with a $5 \times 5 \times 5$ neighbourhood.

We have also investigated the influence of the smoothing parameter $\alpha$ (defined in Section 3.1) on the segmentation results. Fig. 3(b) shows that, in agreement with Buades et al. [4], values of $\alpha$ around 1 provide the best results. Moreover, the graph shows that the algorithm is not sensitive to this parameter if its value is set slightly above 1 ( $\alpha$ is set to 1.1 for the validations in subsection 4.2).

\subsection{Evaluation of the Contribution of the Non-Local Framework}

To evaluate the contribution of the non-local framework to the efficiency of the segmentation process, we have also compared the following versions of FCM:

1. classic FCM $[10]$; 


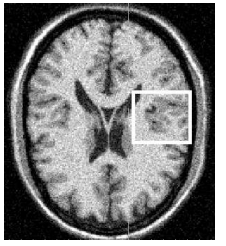

(a)

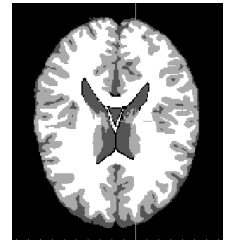

(b)

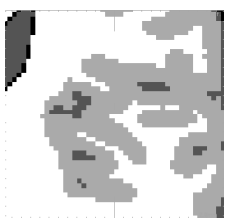

(f)

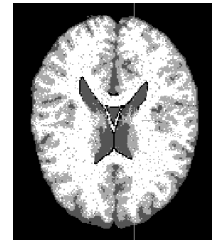

(c)

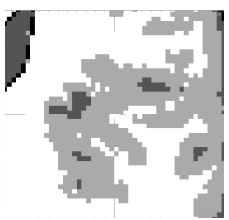

$(\mathrm{g})$

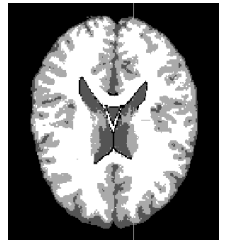

(d)

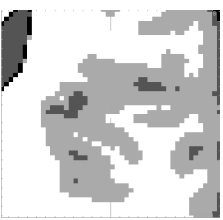

(h)

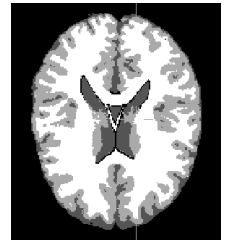

(e)

Fig. 2. Results of segmentations using a T1-weighted image with a $9 \%$ Rician noise. (a) Original image with zoom area, (b) Brainweb's ground truth, (c) simple FCM segmentation, (d) RFCM segmentation, (e) NL-FCM segmentation, (f) zoom on Brainweb's ground truth, (g) zoom on RFCM segmentation, (h) zoom on NLFCM segmentation.

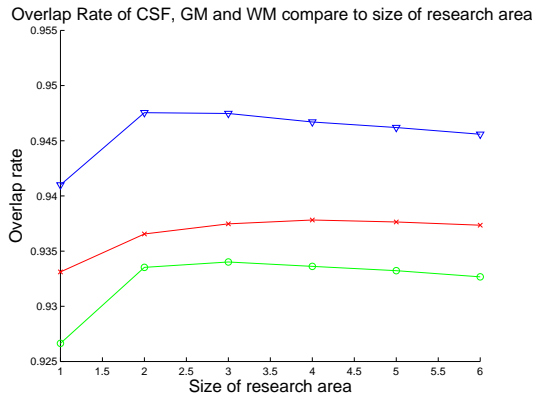

(a)

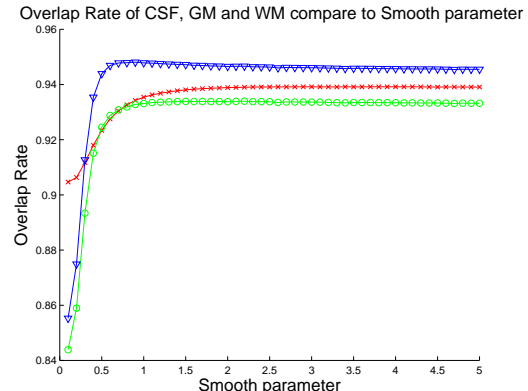

(b)

Fig. 3. Influence of the different parameters. Application on a Brainweb T1-weighted image with $9 \%$ Rician noise. (a) Overlap rate to the Size of Search Area $N_{j}$ and (b) overlap rate to Smooth parameter $\alpha$. Legend: GM (o), WM $(\nabla), \operatorname{CSF}(\times)$.

\begin{tabular}{|l|c|c|c|}
\hline Methods & CSF & GM & WM \\
\hline Classic FCM [10] & 90.4635 & 84.3567 & 85.4812 \\
RFCM without weights [9] & 92.0914 & 91.1193 & 92.9095 \\
RFCM with weights & 92.7614 & 91.0874 & 92.4898 \\
NL-FCM without weights & 92.2247 & 92.2154 & 94.1175 \\
\cline { 2 - 3 } NL-FCM with weights & 93.6307 & 93.3486 & 94.7661 \\
\hline
\end{tabular}

Table 1. Application of different segmentations on a Brainweb T1-weighted image with a $9 \%$ Rician noise. Comparison of the different overlap rates for CSF, GM and WM. 


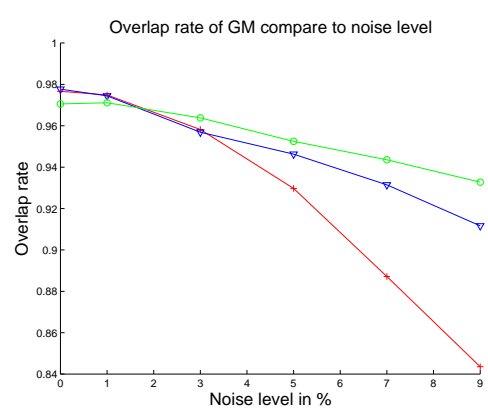

(a)

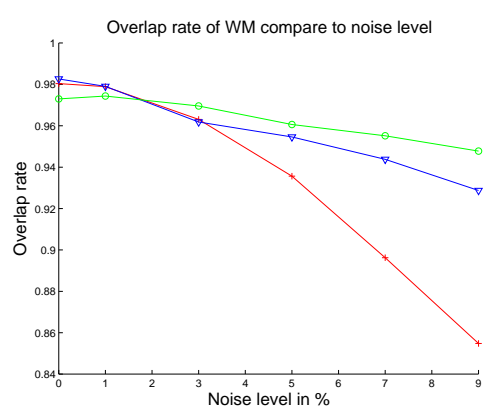

(b)

Fig. 4. Application of different techniques on the same Brainweb T1-weighted image with different noise level. (a) Overlap rate of GM, (b) overlap rate of WM. Legend: NL-FCM (o), RFCM ( $\nabla)$ [9], FCM (+) [10].

2. RFCM [9];

3. RFCM with adaptive weights;

4. NL-FCM with fixed weights; and

5. NL-FCM with adaptive weights.

The results are reported in Table 1. The NL Regularisation approach improves the segmentation results with respect to classic FCM and RFCM. The comparison between RFCM and NL-FCM without weights shows that using a larger neighbourhood leads to significant improvements especially for GM and WM (approx. $1 \%$ ). Moreover, considering extended neighbourhood, introducing NL approach results in a better overlap rate.

Fig. 2 provides a visual insight of these improvements, especially on GM and CSF. This may be due to the low contrast between CSF and GM on a noisy image which can however be correctly handled by the NL regularisation framework. In addition, we observe that NL-FCM results resolve fine structure more clearly such as the borders between ventricles and GM, and around cortical sulci as shown by the zooms done on RFCM segmentation in Fig. $2(\mathrm{~g})$ and on NL-FCM segmentation in Fig. 2(h) compared to the ground truth in Fig. 2(f).

We carried out complementary experiments to determine the robustness to noise for classic FCM [10], RFCM [9] and NL-FCM with Brainweb T1-weighted images with varying noise levels (see Fig. 4). It can be seen that NL-FCM begins to emerge as a strong approach at noise levels of $3 \%$ and above, and becomes more accurate compared to RFCM approach at a $5 \%$ noise.

\section{Conclusion}

In this article, an extension of the Robust Fuzzy C-Means Algorithm method [9] has been proposed, by introducing a non-local approach in the regularisation term, and by considering adaptive (i.e. possibly large) neighbourhoods for 
computing this term. The method depends on parameters which do not require a very fine setting. Experiments performed on several noisy brain MR images (up to $9 \%$ Rician noise) from the Brainweb database emphasise the usefulness of this extension. Additional experiments are also needed to evaluate how the regularisation strength is related to the underlying spatial resolution of the reconstructed imaging data. Overall, this new approach may be particularly useful in more challenging imaging applications such as those limited by the imaging time, for example in imaging the moving human foetus.

\section{Acknowledgement}

The research leading to these results has received funding from the European Research Council under the European Community's Seventh Framework Programme (FP7/20072013 Grant Agreement no. 207667). This work is also funded by NIH Grant R01 NS055064 and a CNRS grant for collaboration between LSIIT and BICG.

\section{References}

1. S.P. Awate, H. Zhang, and J.C. Gee. A fuzzy, non parametric segmentation framework for DTI and MRI analysis: With applications to DTI-tract extraction. IEEE Trans. Med. Imaging, 26(11):1525-1536, 2007.

2. P.-L. Bazin and D.L. Pham. Topology-preserving tissue classification of magnetic resonance brain images. IEEE Trans. Med. Imaging, 26(4):487-496, 2007.

3. S. Bougleux, G. Peyré, and L. Cohen. Non-local regularization of inverse problems. In ECCV'08, Proceedings, Part III, volume 5304 of $L N C S$, pages 57-68. Springer, 2008.

4. A. Buades, B. Coll, and J.M. Morel. A review of image denoising algorithms, with a new one. Multiscale Modeling \& Simulation, 4(2):490-530, 2005.

5. C.A. Cocosco, V. Kollokian, R.K.-S. Kwan, and A.C. Evans. BrainWeb: Online interface to a 3D MRI simulated brain database. In $H B M^{\prime}{ }^{\prime} 7$, Proceedings, volume 5(4 Pt 2) of NeuroImage, page S425, 1997.

6. P. Coupé, P. Yger, S. Prima, P. Hellier, C. Kervrann, and C. Barillot. An optimized blockwise nonlocal means denoising filter for 3-D magnetic resonance images. IEEE Trans. Med. Imaging, 27(4):425-441, 2008.

7. R.K.-S. Kwan, A.C. Evans, and G.B. Pike. MRI simulation-based evaluation of image-processing and classification methods. IEEE Trans. Med. Imaging, 18(11):1085-1097, 1999.

8. M. Mignotte. A non-local regularization strategy for image deconvolution. Pattern Recognition Letters, 29(16):2206-2212, 2008.

9. D.L. Pham. Spatial models for fuzzy clustering. Computer Vision and Image Understanding, 84(2):285-297, 2001.

10. D.L. Pham, J.L. Prince, Dagher A.P., and C. Xu. An automated technique for statistical characterization of brain tissues in magnetic resonance imaging. International Journal of Pattern Recognition and Artificial Intelligence, 11(8):1189-1211, 1996.

11. F. Rousseau. Brain hallucination. In ECCV'08, Proceedings, Part I, volume 5302 of $L N C S$, pages 497-508. Springer, 2008.

12. A.N. Tikhonov. Regularization of incorrectly posed problems. Soviet mathematics. Doklady, 4(6):1624-1627, 1963. 